\title{
Músculo adutor do polegar e força de preensão palmar: potenciais métodos de avaliação nutricional em pacientes ambulatoriais com acidente vascular encefálico
}

\author{
Adductor pollicis muscle and hand grip strength: potential methods of nutritional assessment \\ in outpatients with stroke
}

Débora Rocha Oliveira ${ }^{1}$, Vera Silvia Frangella ${ }^{2}$

\section{RESUMO}

Objetivo: Avaliar e comparar a efetividade de diferentes métodos utilizados para avaliação nutricional de pacientes ambulatoriais hemiplégicos que sofreram acidente vascular encefálico. Métodos: Estudo transversal realizado com pacientes adultos e idosos, de ambos os gêneros, atendidos em um centro de reabilitação. As variáveis analisadas foram medidas antropométricas, bioimpedância, força de preensão palmar e espessura do músculo adutor do polegar. Utilizou-se 0 teste do $\chi^{2}$ de Pearson para verificar a associação entre as variáveis analisadas com nível de significância $\alpha=5 \%$. Resultados: Ao avaliar a associação entre os indicadores de massa muscular, observou-se que a força de preensão palmar em ambos os gêneros foi positivamente correlacionada com a circunferência muscular do braço $(p=0,0196)$ e massa magra ( $p=0,0002)$. Já a massa gordurosa mensurada pelo método de bioimpedância apresentou relação inversa significante com a preensão palmar $(r=-0,3879)$. A espessura do músculo adutor do polegar apresentou associação significante com massa magra ( $p=0,0052$ ) e preensão palmar ( $p=0,0024)$. Conclusão: Neste estudo, a força de preensão palmar e espessura do músculo adutor do polegar foram bem correlacionadas com as mensurações determinadas pela antropometria e pela bioimpedância. Os resultados apontam a aplicabilidade da dinamometria e da espessura do músculo adutor do polegar na prática clínica como métodos de avaliação nutricional para essa população, especialmente idosos, pois detectam alterações funcionais não captadas por outros parâmetros em curto prazo, sendo importantes para identificação precoce do risco nutricional.

Descritores: Acidente cerebral vascular; Avaliação nutricional; Dinamômetro de força muscular; Antropometria; Composição corporal

\section{ABSTRACT}

Objective: To evaluate and compare the effectiveness of different methods used for nutritional assessment of outpatients who had hemiplegic stroke. Methods: A cross-section study with adult and elderly patients of both genders enrolled in a rehabilitation center. The analyzed variables were anthropometric measurements, bioelectrical impedance, hand grip strength and thickness of the adductor pollicis muscle. The Pearson $\chi^{2}$ test was used to check the association between variables with a significance level of $\alpha=5 \%$. Results: When evaluating the association between indicators of muscle mass, it was observed that the hand grip strength in both genders was positively correlated with arm muscle circumference $(p=0.0196)$ and lean mass ( $p=0.0002$ ). Fat mass measured by the bioelectrical impedance method already showed a significant inverse relationship with the grip ( $r=-0.3879)$. The thickness of the adductor pollicis muscle showed significant association with lean mass ( $p=0.0052$ ) and hand grip ( $p=0.0024)$. Conclusion: In this study, the hand grip strength and thickness of the adductor pollicis muscle were well correlated with measurements determined by anthropometry and bioimpedance. The results show the applicability of grip strength and thickness of the adductor pollicis muscle in clinical practice as nutritional assessment methods for this population, especially elderly patients, since they detect functional changes not captured by other parameters in the short term and are important for early identification of risk nutrition.

Keywords: Stroke; Nutrition assessment; Muscle strength; Dynamometer; Anthropometry; Body composition

\section{INTRODUÇÃO}

O acidente vascular encefálico (AVE) pode ser definido como déficit neurológico focal súbito devido a uma lesão vascular, podendo ser do tipo isquêmico ou hemorrágico ${ }^{(1)}$. Segundo a Organização Mundial de

\footnotetext{
Trabalho realizado no Centro Universitário São Camilo, São Paulo (SP), Brasil.

'Pós-graduanda (Mestrado) da Faculdade de Saúde Pública da Universidade de São Paulo - USP; Professora do Curso de Nutrição do Centro Universitário São Camilo - São Paulo (SP), Brasil.

${ }^{2}$ Mestre em Gerontologia; Professora do Curso de Nutrição do Centro Universitário São Camilo, São Paulo (SP), Brasil.

Autor correspondente: Débora Rocha Oliveira - Avenida Nazaré, 1.501 - Ipiranga - CEP 04263-100 - São Paulo (SP), Brasil - Tel.: 11 2588-4044 - E-mail: drocha@saocamilo-sp.br

Data de submissão: 13/1/2010 - Data de aceite: 11/10/2010
} 
Saúde ${ }^{(2)}$, o AVE é a terceira causa de morte em países desenvolvidos, superado apenas pelas doenças coronarianas e câncer. Na população brasileira, o AVE constitui-se como causa principal de mortalidade e incapacidade funcional, acometendo principalmente indivíduos acima de 50 anos, excedendo até mesmo as doenças cardíacas e o câncer ${ }^{(2,3)}$.

As neuropatias agudas levam a um estado hipercatabólico, com rápida perda de massa magra corporal pela mobilização de proteínas de fase aguda e um balanço nitrogenado negativo, podendo levar à subnutrição e, consequentemente, a um acometimento da capacidade funcional ${ }^{(4)}$.

A avaliação do estado nutricional dos pacientes com AVE é, portanto, fundamental para a determinação do diagnóstico, o qual irá nortear a conduta nutricional, a fim de minimizar as taxas de morbimortalidade, além de garantir a qualidade de vida ${ }^{(5)}$.

Existem diversos métodos para a estimativa da composição corporal com diferentes níveis de precisão, custo e aplicabilidade e, recentemente, novos parâmetros vêm sendo incluídos como coadjuvantes na avaliação antropométrica.

Dentre os métodos de avaliação não convencionais, tem-se a força de preensão palmar (FPP), com uso da dinamometria - um método validado para avaliação nutricional - e a mensuração da medida do músculo adutor do polegar (MAP). Ambos são de fácil manuseio, baixo custo, simples e rápidos, permitindo a identificação dos indivíduos em maior risco de desenvolver complicações relacionadas à má nutrição, além de auxiliarem o acompanhamento dos pacientes com subnutrição e com desordens neuromusculares no decurso da investigação clínica ${ }^{(6-9)}$.

Diante do exposto, fica evidente que o indivíduo com AVE apresenta suscetibilidade a déficits no estado nutricional, indicando-se a importância de se estabelecer um diagnóstico nutricional fidedigno com emprego de instrumentos de avaliação seguros, práticos, de fácil aplicabilidade e confiáveis. Assim, o presente estudo visou avaliar e comparar a efetividade de diferentes métodos utilizados para avaliação nutricional de pacientes ambulatoriais hemiplégicos que sofreram AVE.

\section{MÉTODOS}

Trata-se de um estudo transversal, realizado no período de Novembro de 2008 a Março de 2009, com pacientes entre 20 e 59 anos (adultos) e a partir de 60 anos (idosos), sem restrição de gênero, acometidos por AVE isquêmico ou hemorrágico e hemiplégicos. Os pacientes foram atendidos no Centro de Reabilitação Promove São Camilo, situado no município de São Paulo, após a aprovação da Comissão de Ética em Pesquisa do Cen- tro Universitário São Camilo, sob o parecer no 157/08. O tamanho da amostra foi determinado pelo número de atendimentos da clientela caracterizada (durante os cinco meses estipulados para a coleta de dados) que assinou o termo de consentimento livre e esclarecido.

As variáveis avaliadas foram dados antropométricos, exame de bioimpedância (BIA, do inglês bioimpedance analysis), FPP (kgf) e da espessura do MAP (mm).

As mensurações de peso, estatura, circunferência de braço (CB), dobra cutânea triciptal (DCT) e subescapular (DCSE), circunferência muscular do braço (CMB) e MAP (Figura 1) foram obtidas seguindo-se as técnicas padrão ${ }^{(10,11)}$. A bioimpedância foi realizada com aparelho Biodynamics ${ }^{\circledR}$, modelo 310, seguindo-se protocolo do fabricante e dinamometria mensurada através de dinamômetro de mão Jamar ${ }^{\circledR}$, conforme recomendações padrão $^{(12,13)}$ (Figura 2). O peso foi obtido por meio de uma balança de plataforma eletrônica, com capacidade de $500 \mathrm{~kg}$ e escala de $100 \mathrm{~g}$ (Micheletti ${ }^{\circledR}$ ), previamente calibrada. Para a estatura, utilizou-se um estadiômetro

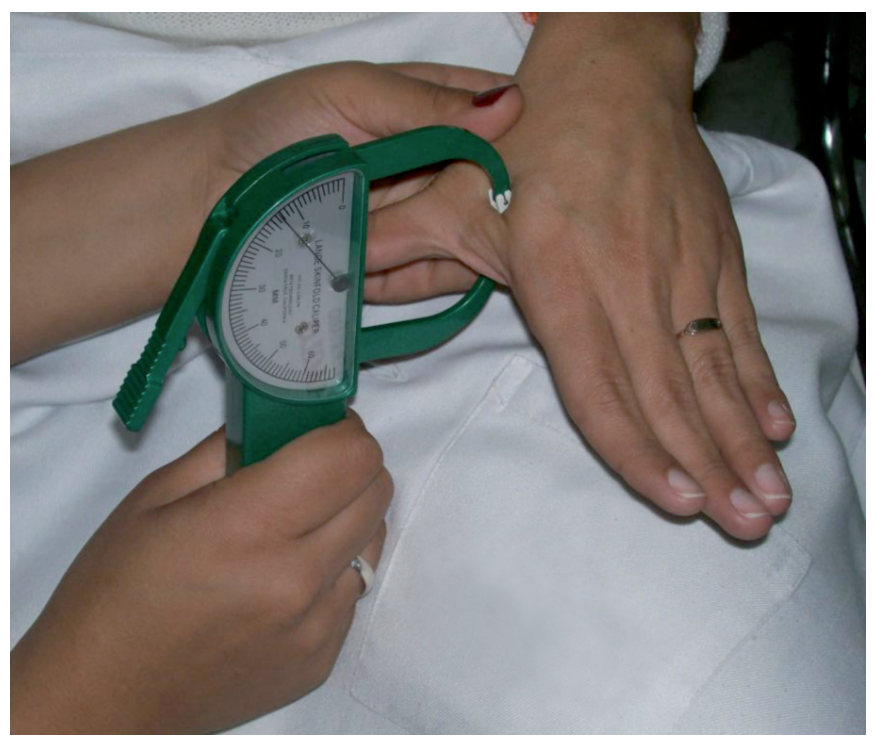

Figura 1. Mensuração da espessura do músculo adutor do polegar

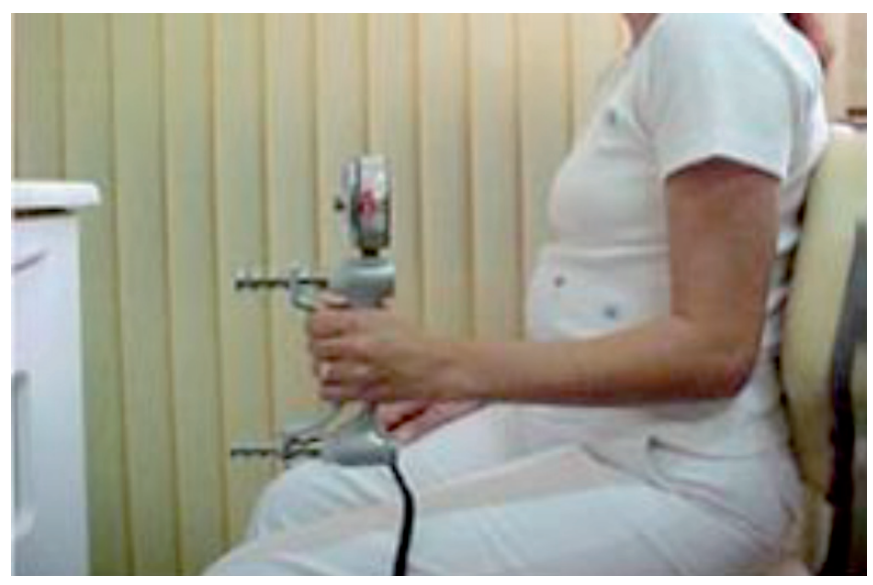

Figura 2. Posição para avaliação da força de preensão palmar 
móvel, graduado em milímetros $\left(\mathrm{Seca}^{\circledR}\right)$. As dobras cutâneas foram verificadas com o auxílio de um plicômetro (Lange ${ }^{\circledR}$ ), com escala de $0,1 \mathrm{~cm}$, devidamente calibrado, sendo cada medida repetida por 3 vezes, anotando-se o valor médio obtido nas medidas ${ }^{(14)}$.

Para a classificação da DCT, CB e CMB nos indivíduos adultos, utilizou-se a classificação percentilar de Frisancho $^{(15,16)}$. Para os idosos, utilizou-se a classificação percentilar de Kuczmarski, Kuczmarski e Najjar ${ }^{(17)}$ para DCT, CMB e CB.

O ponto de corte para classificação da espessura do MAP foi determinado segundo preconizado por Andrade e $\mathrm{Lameu}^{(7)}$, que apontaram valor acima de $14 \mathrm{~mm}$ da espessura do músculo adutor como preditivo negativo para complicações sépticas, não sépticas e mortalidade.

Para análise dos resultados do exame de BIA, foram utilizadas as recomendações descritas no impresso emitido pelo aparelho de bioimpedância, considerando-se a determinação dos valores alvos de cada variável avaliada.

As diferenças entre os gêneros e faixa etária foram determinadas pelo o teste $t$ de Student. O teste do $\chi^{2}$ de Pearson foi utilizado para se verificar a associação entre as variáveis analisadas. O nível de significância adotado em todas as análises foi de $\alpha=5 \%$. As informações estatísticas foram obtidas com o auxílio do programa estatístico GraphPad Prism versão 3.00 para Windows (GraphPad Software).

\section{RESULTADOS}

Avaliaram-se 26 indivíduos adultos e idosos (15 homens e 11 mulheres) com média de idade para ambos os gêneros sem diferença estatística $-48,6 \pm 2,97$ anos para adultos e 70,1 $\pm 2,21$ anos para idosos.
No presente estudo, procurou-se verificar a efetividade dos diferentes métodos de avaliação. Assim, a BIA foi utilizada como padrão de referência para as variáveis determinantes de massa magra e massa gorda. Os valores de correlação (coeficiente r) e de significância (p) das variáveis avaliadas no estudo estão apresentados no Quadro 1

A FPP (Figura 3) foi positivamente correlacionada com os indicadores de estado nutricional, em ambos os gêneros, com exceção do índice de massa corpórea (IMC). Este, por sua vez, apresentou forte associação com CB, DCT e CMB (Figura 4).

Ao avaliar a associação entre os indicadores de massa muscular, observou-se que a massa magra estimada pelo método da BIA apresentou forte correlação com o indicador antropométrico CMB ( $\mathrm{p}=0,0001)$ e com FPP $(p=0,002)$ (Figura 5). Em relação à massa gordurosa, houve correlação positiva entre a porcentagem de gordura corporal (\%GC) mensurada por meio de BIA e DCT $(\mathrm{p}<0,0001)$ e relação inversa com a preensão palmar $(r=-0,3879$ e $p=0,0052)$ (Figura 6).

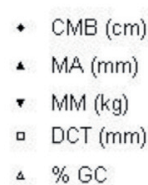

CMB: circunferência muscular do braço; MA: Músculo adutor; MM: massa magra; DCT: dobra triciptal do braço; \%GC: porcentagem de gordura corporal.

Figura 3. Correlação da força de preensão palmar com variáveis antropométricas.

Quadro 1. Correlação de Pearson entre as variáveis avaliadas no estudo

\begin{tabular}{|c|c|c|c|c|c|c|c|c|}
\hline Variáveis & $\begin{array}{c}\text { IMC } \\
\text { Coeficiente } r^{*}\end{array}$ & $\begin{array}{c}\text { CB } \\
\text { Coeficiente } r^{*}\end{array}$ & $\begin{array}{c}\text { CMB } \\
\text { Coeficiente } r^{*}\end{array}$ & $\begin{array}{c}\text { DCT } \\
\text { Coeficiente } r^{*}\end{array}$ & $\begin{array}{l}\text { Massa magra } \\
\text { (BIA) } \\
\text { Coeficiente } r^{*}\end{array}$ & $\begin{array}{c}\% G C \text { (BIA) } \\
\text { Coeficiente } r^{*}\end{array}$ & $\begin{array}{c}\text { Preensão } \\
\text { palmar } \\
\text { Coeficiente } \mathbf{r}^{*}\end{array}$ & $\begin{array}{c}\text { Espessura do } \\
\text { MAP } \\
\text { Coeficiente } r^{*}\end{array}$ \\
\hline IMC & - & $0,8787^{* *}$ & $\begin{array}{c}0,5380 \\
(0,0046)\end{array}$ & $0,6973^{* *}$ & 0,3238 & $0,6570 * * *$ & $-0,05243$ & 0,1074 \\
\hline CB & $0,8707^{* *}$ & - & $0,7377^{* *}$ & $0,6654^{* * *}$ & $0,3999 * * *$ & $0,5044^{* * *}$ & 0,01909 & 0,1888 \\
\hline СMB & $\begin{array}{c}0,5380 \\
(0,0046)\end{array}$ & $0,7377^{* *}$ & - & $\begin{array}{l}-0,01308 \\
(0,9494)\end{array}$ & $0,6823^{* *}$ & $-0,04323$ & $0,4546^{* * *}$ & $0,4618^{* * *}$ \\
\hline $\begin{array}{l}\text { Massa magra } \\
\text { (BIA) }\end{array}$ & 0,3238 & $0,3999 * * *$ & $0,6823^{* *}$ & $-0,1617$ & - & $-0,3594$ & $0,6639 * * *$ & $0,5315^{* * *}$ \\
\hline$\%$ GC (BIA) & $0,6570^{* * *}$ & $0,5044^{* * *}$ & $-0,04323$ & $0,7969^{* *}$ & $-0,3594$ & - & $-0,6504^{* * *}$ & $-0,3879 * * *$ \\
\hline $\begin{array}{l}\text { Preensão } \\
\text { palmar }\end{array}$ & $\begin{array}{l}-0,05243 \\
(0,7992)\end{array}$ & 0,01909 & $0,4546^{* * *}$ & $-0,4744^{* * *}$ & $0,6639 * * *$ & $-0,6504^{* * *}$ & - & $0,5697^{* * *}$ \\
\hline Espessura MAP & 0,1074 & 0,1888 & $0,4618^{* * *}$ & $-0,2313$ & $0,5315^{* * *}$ & $-0,3879 * * *$ & $0,5697 * * *$ & - \\
\hline
\end{tabular}

IMC: índice de massa corpórea; CB: circunferência do braço; CMB: circunferência muscular do braço; DCT: dobra triciptal do braço; BIA: bioimpedância; \%GC: porcentagem de gordura corporal; MAP: músculo adutor do polegar. ${ }^{*}$ r: correlação de Pearson; ** $p<0,0001 ;{ }^{* * *} p<0,05$ 
- $\mathrm{CMB}(\mathrm{cm})$

- $\mathrm{CB}(\mathrm{cm})$

- DCT (mm)

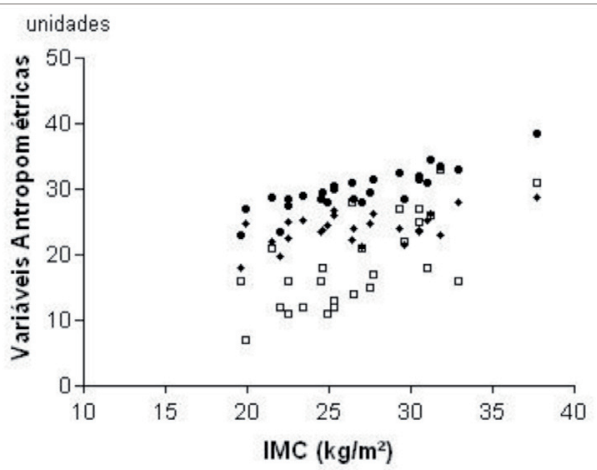

CMB: circunferência muscular do braço; CB: circunferência do braço; DCT: dobra triciptal do braço.

Figura 4. Correlação do índice de massa corpórea (IMC) com variáveis antropométricas

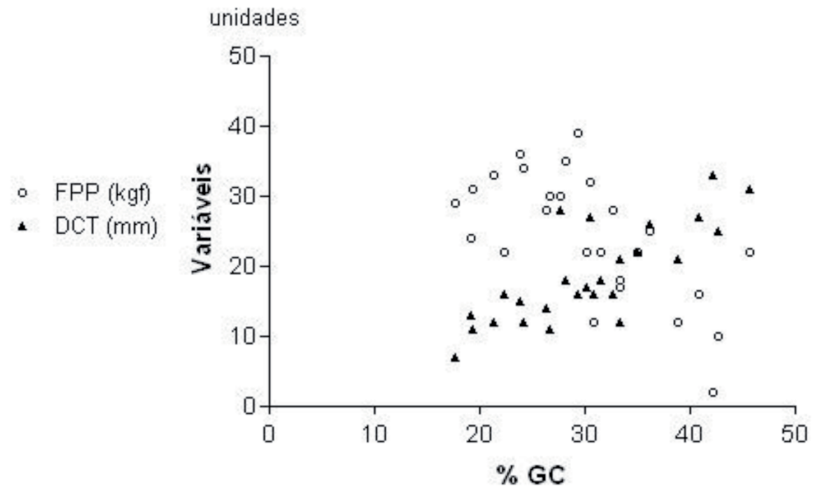

Figura 6. Correlação da porcentagem de gordura corporal (\%GC) com força de preensão palmar (FPP) e dobra cutânea triciptal (DCT)

A \% GC foi fortemente correlacionada com IMC e DCT e apresentou relação inversa com FPP. Os valores da espessura do MAP apresentaram correlação positiva com a massa magra $(\mathrm{p}=0,0052)$ e FPP $(\mathrm{p}=$ 0,0024) (Figura 7).

\section{DISCUSSÃO}

Atualmente, muito se tem discutido sobre os diferentes instrumentos de avaliação nutricional, e o IMC é apresentado geralmente com destaque por ser um método de fácil aplicação, baixo custo e não invasivo ${ }^{(18,19)}$. Contudo, apesar de neste estudo o IMC também ter apresentado forte correlação com os indicadores antropométricos, não foi capaz de distinguir a composição corporal e não expressou a distribuição da gordura e massa magra corporal. Dessa forma, o IMC não pode ser utilizado como única estimativa de obesidade ou massa corporal gorda.

Diversos estudos sustentam o fato de que a FPP é positivamente associada com estado nutricional ${ }^{(20-22)}-$ dados que corroboram os achados desta pesquisa.

Similarmente, um estudo realizado com 94 indivíduos adultos e idosos da zona rural do Malawi, para ava-

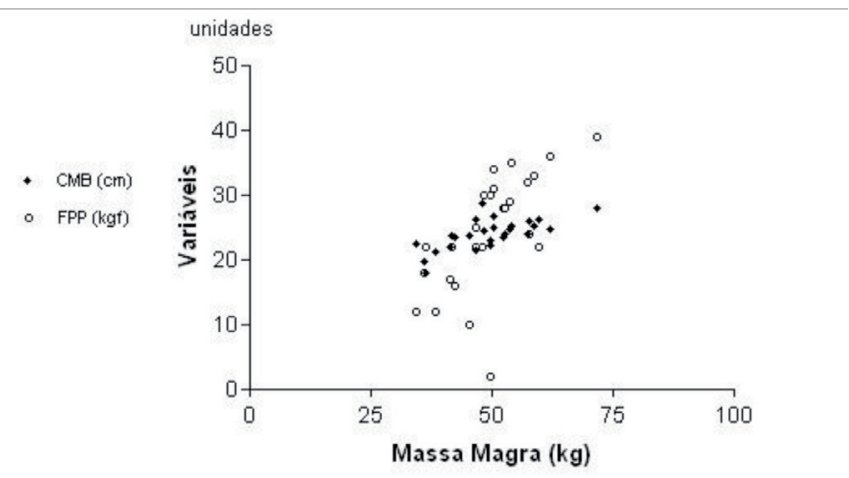

Figura 5. Correlação da massa magra determinada pela bioimpedância com força de preensão palmar (FPP) e circunferência muscular do braço (CMB)

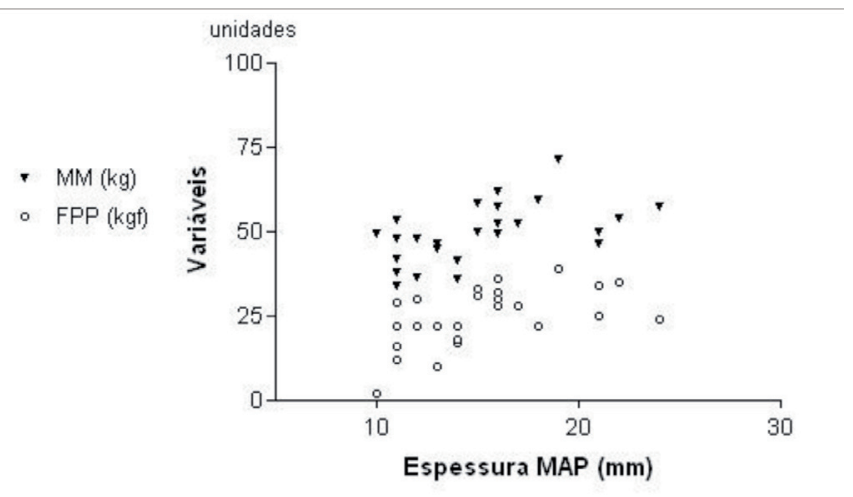

Figura 7. Correlação da espessura do músculo adutor do polegar (MAP) com força de preensão palmar (FPP) e massa magra (MM)

liar a relação entre estado nutricional e FPP apresentou forte correlação do aumento da FPP com aumento de massa magra ${ }^{(23)}$.

Relação inversa também foi encontrada por Hulens et al. ${ }^{(24)}$, que observaram menor força muscular periférica nas mulheres obesas quando comparadas às eutróficas, apresentando correlação negativa entre FPP e \%GC.

Além de indicador de estado nutricional, a dinamometria também é bastante referenciada como preditora de mortalidade, independentemente do IMC. É um método de avaliação muito utilizado para avaliar força muscular da mão e um ótimo preditor de prognóstico de complicações e saúde ${ }^{(25-28)}$.

$\mathrm{O}$ aspecto da perda de massa magra pode ser estimado pela FPP. Neste estudo, a FPP foi bem correlacionada com as mensurações determinadas pela antropometria e pela BIA. Assim, legitima-se o fato de que a FPP é positivamente associada com estado nutricional ${ }^{(20-22)}$.

No Reino Unido, em estudo com indivíduos saudáveis para avaliar a relação entre as medidas estruturais e funcionais e o estado nutricional encontrou forte associação da preensão palmar com $\mathrm{CMB}^{(29)}$. Resultados semelhantes foram encontrados no presente estudo. 
A massa magra e a força muscular são determinantes para funcionalidade em idosos. Arroyo et al. ${ }^{(27)}$, ao verificarem os indicadores antropométricos e composição corporal com funcionalidade em 377 idosos chilenos, encontraram correlação negativa entre limitação funcional e FPP em ambos os gêneros. Essa diminuição de massa magra e de força muscular induzida pelo envelhecimento também é documentada por Landers et al. ${ }^{(30)}$.

Outro instrumento para avaliação nutricional que vem recebendo atenção é a espessura do MAP - um método simples que proporciona a avaliação de prognósticos para complicações hospitalares, assim como auxilia no diagnóstico nutricional ${ }^{(11)}$. Nossos achados também evidenciaram associação positiva da massa magra determinada pela BIA e a FPP com a espessura do MAP. A redução da espessura do MAP ocorre progressivamente à medida que as atividades diárias são substancialmente reduzidas pela apatia induzida pela desnutrição e envelhecimento ${ }^{(7)}$.

Cabe salientar também que a FPP, além de auxiliar na avaliação do estado nutricional e nortear a conduta dietética, é um instrumento importantíssimo no ambiente hospitalar por ser preditor de mortalidade e complicações clínicas.

Outro instrumento que merece destaque (recomendando-se sua inclusão nos métodos de avaliação nutricional) é a mensuração da espessura do MAP; além de apresentar importante associação com indicadores de massa muscular, é um método de avaliação simples, direto e sem necessidade de cálculos, sendo capaz de indicar risco para desnutrição e prognósticos para complicações hospitalares e risco de desnutrição, reduzindo, assim, os custos hospitalares e a mortalidade.

\section{CONCLUSÃO}

Diante da forte associação entre estado nutricional e FPP, sugere-se que a dinamometria seja incluída na prática clínica como um método de avaliação nutricional, uma vez que a FPP é capaz de detectar alterações funcionais que não são captadas por outros parâmetros em curto prazo, sendo importante para identificação precoce de indivíduos em risco nutricional e para o acompanhamento das intervenções e evolução clínica, além de ser um método de fácil manuseio, rápido, de baixo custo e não invasivo.

\section{REFERÊNCIAS}

1. Andre C. Manual de AVC. 2 ed. Rio de Janeiro: Revinter, 2006.

2. Mackay J, Mensah GA, Mendis S, Greenlund K. The atlas of heart disease and stroke. Genebra: World Health Organization; 2004.
3. Nitrini R, Bacheschi LA. A Neurologia que todo médico deve saber. São Paulo: Ateneu:2003.

4. Dávalos A, Ricart W, Gonzalez-Huix F, Soler S, Marrugat J, Molins A, et al. Effect of malnutrition after acute stroke on clinical outcome. Stroke. 1996;27(6):1028-32.

5. Batista MCR, Franceschini SCC, Priore SE. Avaliação de indicadores antropométricos de adultos e idosos brasileiros. Rev Soc Bras Aliment Nutr. 2002;23(1):67-78.

6. Luna-Heredia E, Martín-Peña G, Ruiz-Galiana J. Handgrip dynamometry in healthy adults. Clin Nutr. 2005;24(2):250-8.

7. Andrade PV, Lameu EB. Espessura do músculo adutor do polegar: um novo indicador prognóstico em pacientes clínicos. Rev Bras Nutr Clin.2007;22(1):2835.

8. Moreira D, Álvarez RRA, Gogoy JR, Cambraia AN. Abordagem sobre preensão palmar utilizando o dinamômetro $\mathrm{JAMAR}^{\circledR}$ : uma revisão de literatura. R Bras Cienc Mov. 2003;11(2):95-9.

9. Carlo MM, Luzo MC. Terapia ocupacional: reabilitação física e contextos hospitalares. São Paulo: Roca; 2004.

10. Duarte AC, Castellani FR. Semiologia nutricional. Rio de Janeiro: Axcel Books; 2002.

11. Andrade FN, Lameu EB, Luiz RR. Musculatura adutora do polegar: um novo índice de prognóstico em cirurgia cardíaca valvar. Rev SOCERJ. 2005;18(5):384-91.

12. Moreira $D$, Álvares RR. Avaliação da força de preensão palmar com o uso do dinamômetro Jamar ${ }^{\circledR}$ em pacientes portadores de hanseníase atendidos em nível ambulatórios no Distrito Federal. Hansen Int. 2002;27(2):61-9.

13. D'Oliveira GD. Avaliação funcional da força de preensão palmar com dinamômetro Jamar $^{\circledR}$ : estudo transversal de base populacional [tese]. Brasília: Universidade Católica de Brasília, Brasília; 2005. [citado 2008 Jun 11]. Disponível em: < http://www.ucb.br/mestradoef/Dissertacoes/2005/Georgia\%20Danila\%20 Fernandes\%20DOliveira/Disserta\%C3\%A7\%C3\%A30\%20Final-\%20Georgia.pdf >

14. Gibson RS. Principles of Nutritional Assessment. 2nd ed. New York: Oxford; 2005.

15. Frisancho AR. New norms of upper limb fat and muscle areas for assessment of nutritional status. Am J Clin Nutr. 1981;34(11):540-5.

16. Frisancho AR. Anthropometric Standards for the Assessment of Growth and Nutritional Status. 4th ed. Ann Arbor, MI, USA: University of Michigan; 1990.

17. Kuczmarski MF, Kuczmarski RJ, Najjar M. Descriptive anthropometric reference data for older Americans. J Am Diet Assoc. 2000;100(1):59-66.

18. Anjos LA. Índice de massa corporal (massa corporal.estatura-2) como indicador do estado nutricional de adultos: revisão da literatura. Rev Saúde Pública. 1992; 26(6):431-6.

19. Landi F, Onder G, Gambassi G, Pedone C, Carbonin P, Bernabei R. Body mass index and mortality among hospitalized patients. Arch Intern Med. 2000;160(17):2641-4.

20. Cereda E, Vanotti A. Short dietary assessment improves muscle dysfunction identification by Geriatric Nutritional Risk Index in uncomplicated institutionalised patients over 70 years old. Clin Nutr. 2008;27(1):126-32.

21. Pieterse $S$, Manandhar $M$, Ismail $S$. The association between nutricional status and handgrip strength in older Rwandan refugees. Eur J Clinl Nutr. 2002;56(10):933-9.

23. Chilima DM, Ismail SJ. Nutrition and handgrip strength of older adults in rural Malawi. Public Health Nutr. 2000;4(1):11-7.

22. Barbosa AR, Souza JMP, Lebrão ML, Marucci MF. Relação entre estado nutricional e força de preensão palmar manual em idosos do município de São Paulo, Brasil: dados da pesquisa SABE. Rev Bras Cineantropom Desempenho Hum. 2006;8(1):37-44.

24. Hulens M, Vansant G, Lysens R, Claessens AL, Muls E, Brumagne S. Study of differences in peripheral muscle strength of lean versus obese women: an allometric approach. Int J Obes Relat Metab Disord. 2001;25(5):676-81. 
25. Rantanen T, Harris T, Leveille SG, Visser M, Foley D, Masaki K, et al. Muscle strength and body mass index as long-term predictors of mortality in initially healthy men. J Gerontol A Biol Sci Med Sci. 2000;55(3):M168-73.

26. Newman AB, Kupelian V, Visser M, Simonsick EM, Goodpaster BH, Kritchevsky $\mathrm{SB}$, et al. Strength, but not muscle mass, is associated with mortality in the health, aging and body composition study cohort. J Gerontol A Biol Sci Med Sci. 2006;61(1):72-7.

27. Arroyo P, Lera L, Sánchez H, Bunout D, Santos JL, Albala C. Indicadores antropométricos, composición corporal y limitaciones funcionales en ancianos. Rev Méd Chile, 2007;135(7):846-54.
28. Gale CR, Martyn CN, Cooper C, Sayer AA. Grip stregth, body composition, and mortality. Intern J Epidemiol. 2007;36(1):228-35.

29. Hornby ST, Nunes QM, Hillman TE, Stanga Z, Neal KR, Rowlands BJ, et al. Relationships between structural and functional measures of nutritional status in a normally nourished population. Clin Nutr. 2005;24(3):421-6.

30. Landers KA, Hunter GR, Wetzstein CJ, Bamman MM, Weinsier RL. The interrelationship among muscle mass, strength, and the ability to perform physical tasks of daily living in younger and older women. J Gerontol A Biol Sci Med Sci. 2001;56(10):B443-8. 